\title{
The Habitability of Circumbinary Planets: Stellar and Planetary Interactions
}

\author{
Paul A. Mason \\ New Mexico State University - DACC, Las Cruces, NM, 88003, USA \\ E-mail:pmason@nmsu.edu
}

Jorge I. Zuluaga

Harvard-Smithsonian Center for Astrophysics, Cambridge, MA 02138, USA

FACom - Instituto de Física - FCEN, Universidad de Antioquia, Calle 70 No. 52-21, Medellín, Colombia

E-mail: jorge.zuluaga@udea.edu.co

\section{Pablo A. Cuartas-Restrepo \\ FACom - Instituto de Física - FCEN, Universidad de Antioquia, Calle 70 No. 52-21, Medellín, Colombia \\ E-mail: pablo.cuartas@udea.edu.co}

Efforts to study the potential habitability of planets around moderately close binaries are presented. With this aim stellar and planetary interactions are investigated, focusing on their impact on habitability. We provide a comprehensive, web-based, BHM Calculator (http://bhmcalc.net), capable of exploring the large parameter space offered by binaries and circumbinary planets. After estimating the limits of the instantaneous and continuous habitable zone for any main sequence binary plus planet configuration, surface conditions for photosynthesis as compared to Earth are calculated. Key properties for habitability, namely XUV and stellar wind flux, are calculated as proxies to investigate planets located at any distance beyond a critical limit for dynamical stability. Stellar rotational evolution is of critical importance for habitability. It involves all aspects of single star evolution plus mutual gravitational tides. The stars apply a torque on each other with the binary orbit as the driving frequency. Collectively, the potential benefits that circumbinary planets have over single stars is called the "Binary Habitability Mechanism" (BHM).

Models are applied to four mock Earth-like planets orbiting in the circumbinary habitable zone of different binaries. These are chosen for illustration as they are interesting niches for life. We suggest that stellar and planetary interactions are such that an Earth-like planet (or even in some cases a Mars-like planet or moon) may experience conditions that could be conducive to moist habitable environments.

Frontier Research in Astrophysics,

26-31 May 2014

Mondello (Palermo), Italy 


\section{Introduction}

The outstanding work of the Kepler Observatory circumbinary team has resulted in the discovery of planetary systems around 8 moderately separated binaries (periods in the range of 8-60 days), each composed of main sequence stars. These are Kepler-16 b (4), Kepler-34 b and Kepler35 b (31), Kepler-38 b (25), Kepler-47 b, c (26) and d (9), Kepler-64 b (PH1) (29; 18), Kepler-413 b (17), and KIC 9632895 b (32). Questions concerning radiation conditions planets actually face while orbiting two stars, have resulted in several studies $(21 ; 27 ; 22 ; 12 ; 6 ; 23 ; 28 ; 35)$. Our approach, as others, is to use the models of (13), updated by (16) derived for single stars to estimate the Circumbinary Habitable Zone (hereafter BHZ) limits. We also investigate the high-energy radiation and plasma environments of the $\mathrm{BHZ}$.

The Kepler telescope is most sensitive to the detection of large planets orbiting small stars with short period orbits. The fact that on average circumbinary planets have longer periods than Kepler planets around single stars is not a selection effect. Short period planets are limited by dynamical stability $(7 ; 10)$. However, circumbinary planets in the BHZ appear to be common as three of the ten known transiting circumbinary planets in the BHZ and three of the eight binaries (38\%), namely Kepler 16, Kepler 47, and KIC 9632895, host (giant) planets in the circumbinary habitable zone (BHZ). See (35) for an analysis of the three Kepler binaries with planets in the BHZ.

This paper is organized as follows. In Section 2, the main characteristics of the Binary Habitability Mechanism are summarized; including the operation of the BHM Calculator, Section 2.1, and its estimations of the instantaneous and continuous BHZ in Section 2.2, as well as isolation and photosynthetic photon flux density (PPFD) in Section 2.3. The study of stellar aggression towards BHZ planets in the sample niches are given in Section 2.4. Finally, we conclude, in Section 3, by discussing results within an astrobiological context. While it is difficult to say at this time how frequently life may exist on circumbinary planets, there are reasons to be confident of the quality of life promoting conditions that may exist on some circumbinary planets as the result of the BHM.

\section{Binary Habitability Mechanism}

In order to characterize conditions in the habitable zones of the Kepler binaries and other model binaries we introduced the so-called "Binary Habitability Mechanism", BHM $(22 ; 23 ; 35)$. Originally the BHM was proposed as the process by which many circumbinary habitable zone planets experience reduced stellar aggression and thereby have the potential to retain water, due to tidal evolution. In some cases, even Mars-like planets may retain a thick atmosphere. The mechanism has evolved to be a combination of several favorable factors found only in binaries, as compared to single stars. These factors include, but are not restricted to, the fact that $\mathrm{HZ}$ zones are located farther in binaries and may be much wider than in single stars. BHZs may allow eccentric planets and possibly multiple planets. Critically, tidal torquing among the stellar components, especially for certain binary periods and initial eccentricities, aids in the reduction of stellar activity. Photosynthetic organisms may also benefit from the presence of two sources of photons instead of one, by taking advantage of often very different spectral ranges while maintaining habitable insolation conditions. The primary mass sets the habitability lifetime and provides the dominant source of radiation. 
BHM thus potentially improves adverse conditions found as the result of stellar and planetary interaction in single stars. Many binaries with moderate orbital periods ( 8 days $<P_{\text {bin }}<60$ days) have wide habitable zones. Many low mass stars, e.g. $M<0.5 M_{\odot}$, may not be able to support life as single stars, yet binaries containing these low mass stars may harbor a habitable planet. A variety of constraints are involved depending especially on stellar masses, binary periods and eccentricities. Conditions are most favorable if tidal torques pre-maturely "age" one or both of the stars. With early rotational braking, stars undergo low magnetic activity and thereby have low aggression towards planets as compared to planets orbiting single stars. For main sequence binaries, the larger star will synchronize first. For purposes of calculation the stars are assumed to be formed at the same time.

Here, detailed results for four model binaries harboring a single hypothetical circumbinary planet in the BHZ are presented. Twelve of these circumbinary niches were presented in (23), but most were not discussed in detail. The four niches described here are Niches 4, 6, 8, and 11, from that paper, see Table 1). Stellar and planetary interaction on an Earth-like planet analogue located at a given place in the $\mathrm{BHZ}$ is examined for each niche. Although no evidence exists yet for small planets or moons in the BHZ, the analysis of model conditions could shed light habitability in actual binaries.

\subsection{Selected Niches}

In Table 1, input properties of the four model BHZ planets and their host stars are listed. Unlike the presentation of these niches in (23), here we have also selected specific planet orbits in order to illustrate several interesting effects. The question addressed here is that if similar real binaries contain an Earth-like planet, then would that planet be habitable based on our understanding of Earth-like habitability?

In order to provide the reader an opportunity to reproduce this results and explore BHM parameter space, we provide an on-line tool, the BHM Calculator ${ }^{1}$. Other BHZ calculators are available, including (3) and (24). The BHM Calculator provides renditions of the instantaneous and continuous BHZ. In addition, it calculates BHM properties of the system including those related to the rotational evolution of the stellar components, the insolation, and the combined XUV and SW fluxes as measured for planets located at different distances from the binary as well the stellar evolution results that are used in the calculations.

The aim of the calculator in general and this paper in particular, is to characterize the radiation and plasma environment of model planets. The BHM Calculator provides all of calculations presented here as well as any other desired binary plus planet configuration. After calculating stellar evolution for both stars, it determines the instantaneous and continuous BHZ. It determines the time variable insolation and PPFD incident at the top of the atmosphere of the planet. Habitability factors, such as the XUV flux, stellar wind flux, and the resulting potential atmospheric erosion for an Earth-like planet in these binaries is estimated. For brevity, we do not include examples of all the results provided by the calculator.

The essence of BHM is that while stars naturally lose angular momentum as they age, mainly due to magnetically induced mass loss (hereafter called magnetic braking), they also may have their

\footnotetext{
${ }^{1}$ http://bhmcalc.net
} 


\begin{tabular}{lcccc}
\hline \hline & Niche 4 & Niche 6 & Niche 8 & Niche 11 \\
\hline $\mathrm{P}_{\text {bin }}(\mathrm{d})$ & 15 & 12 & 20 & 30 \\
$\mathrm{a}_{\mathrm{bin}}(\mathrm{AU})$ & 0.142 & 0.119 & 0.167 & 0.204 \\
$\mathrm{e}_{\mathrm{bin}}$ & 0.4 & 0.1 & 0.3 & 0.4 \\
$\mathrm{M}_{1}\left(M_{\odot}\right)$ & 1.0 & 1.0 & 0.85 & 0.70 \\
$\mathrm{M}_{2}\left(M_{\odot}\right)$ & 0.70 & 0.55 & 0.70 & 0.55 \\
$\mathrm{R}_{1}\left(R_{\odot}\right)$ & 1.02 & 1.02 & 0.759 & 0.650 \\
$\mathrm{R}_{2}\left(R_{\odot}\right)$ & 0.650 & 0.546 & 0.650 & 0.546 \\
$\mathrm{~T}_{\text {eff }}(\mathrm{K})$ & 5910 & 5910 & 5212 & 4461 \\
$\mathrm{~T}_{\text {eff }}(\mathrm{K})$ & 4461 & 3732 & 4461 & 3729 \\
$\mathrm{P}_{\mathrm{p}}(\mathrm{d})$ & 373.18 & 434.83 & 205.99 & 203.75 \\
$\mathrm{a}_{\mathrm{p}}(\mathrm{AU})$ & 1.10 & 1.30 & 0.79 & 0.73 \\
$\mathrm{e}_{\mathrm{p}}$ & 0.0167 & 0.0167 & 0.0167 & 0.0167 \\
$\mathrm{CHZ}_{\text {in }}(\mathrm{AU})$ & 1.24 & 1.20 & 0.72 & 0.71 \\
$\mathrm{CHZ}_{\text {out }}(\mathrm{AU})$ & 1.71 & 1.62 & 1.33 & 0.88 \\
$\mathrm{CHZ}_{\text {width }}(\mathrm{AU})$ & 0.47 & 0.42 & 0.61 & 0.17 \\
\hline \hline
\end{tabular}

Table 1: Circumbinary habitable niches discussed herein. Stellar properties are calculated from stellar models at an adopted age of the Earth of 4.568 Gyr.

magnetic activity evolution modified by the presence of a binary companion (i.e. tidal braking). The dominant frequency in the tidal interaction is that of the binary orbital cycle. So, if the stars are close enough to mutually (tidally) alter their rotation and hence stellar activity evolution then the level and duration of intense coronal XUV emission and stellar winds will be also affected $(22 ; 35)$.

For short period binaries, $P_{\text {bin }} \sim 8$ days, tidal evolution leads to a "forever young" effect, where the stellar dynamo operates vigorously due to high stellar rotation rates as the spins migrate towards the binary frequency. Coronal activity remains at a high level, potentially eroding the atmosphere from planets even for those within the BHZ. Such short period binaries are prime examples for the need to extend habitability concepts beyond the classical habitable zone. On the other hand, the stellar aggression could be minimized by BHM effects in the $\mathrm{BHZ}$ of binaries having certain periods and eccentricities. Kepler 34, Kepler 35, and Kepler $47(22 ; 35)$ are good examples of binaries with enhanced habitability conditions via BHM.

The effect of XUV and stellar wind flux is highly dependent on not only the masses of the stars $M_{1}$ and $M_{2}$, but also on the binary period, $P_{b i n}$, and binary eccentricity, $e_{b i n}$, as the tidal evolution sensitively depends on all of these parameters. The XUV flux and stellar wind aggression of the binary is a function of the mass of the stars (assumed to be on the main sequence) and their rotation rates which correlate with magnetic activity levels. In order to account for all of these effects, the interaction among the binary components, as they age, must be properly modeled. Details of the models used for all of the calculations given here may be found in (35), therefore only results are summarized here.

\subsection{Circumbinary Habitable Zones}

For details concerning our BHZ calculations the reader is referred to (22). In Figure 1, the instantaneous habitable zone is shown for the four binaries with planets listed in Table 1. Stellar 
and planetary parameters as well as habitability conditions are calculated for a planet with same mass, radius, and atmosphere as present day Earth.
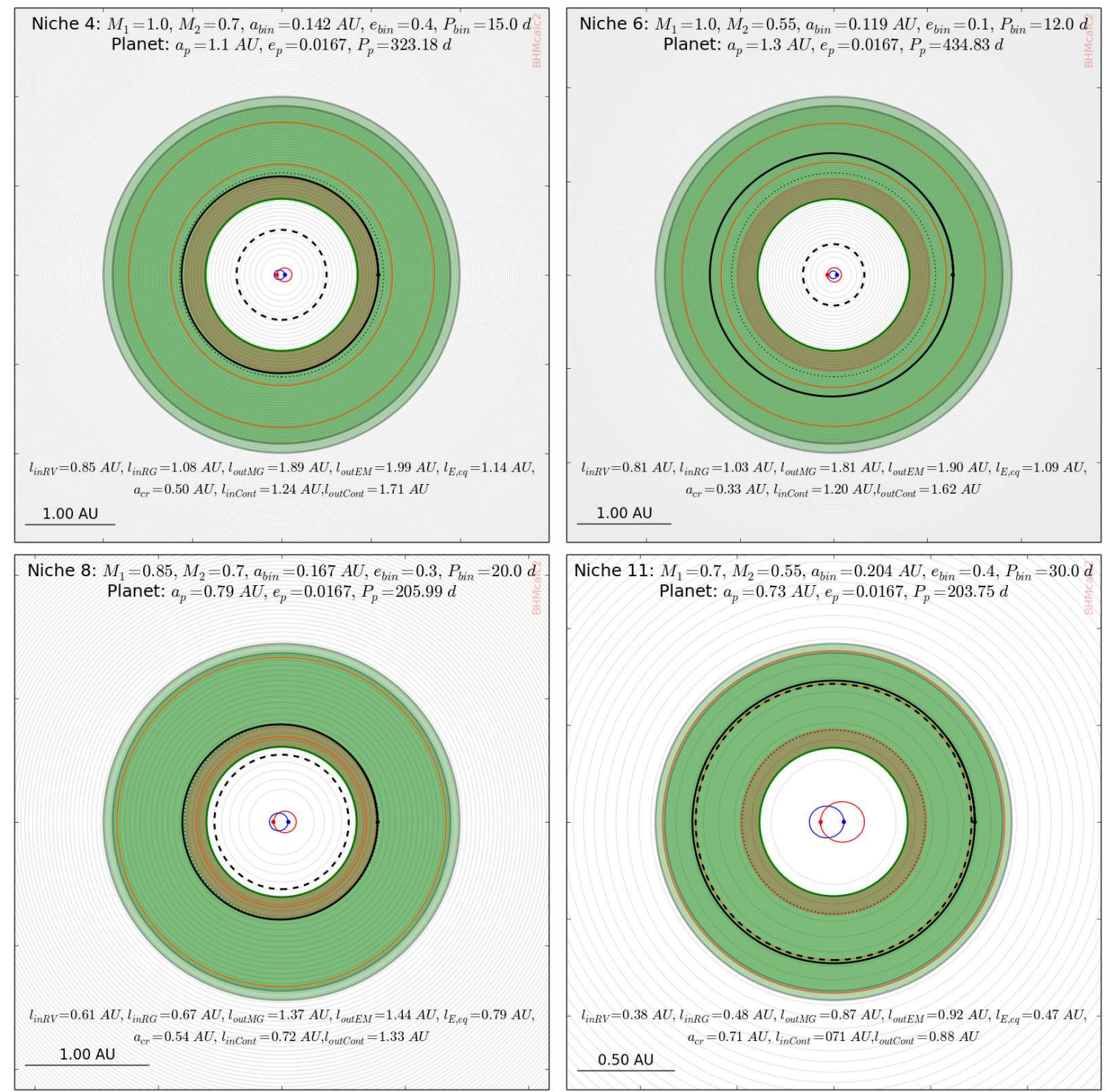

Figure 1: Habitable zones for the hypothetical four binaries discussed herein are shown. The dashed line is the stability limit for planet orbits. The inner HZ limits are 1) the recent Venus (RV) limit and 2) the runaway greenhouse (RG), while the outer limits are 3) the maximum greenhouse (MG) and 4) the early Mars (EM) limit. The age is 4.568 Gyr in each case. Top Left: Niche 4 - Top Right: Niche 6 - Bottom Left: Niche 8 - Bottom Right: Niche 11. This pattern continues for all subsequent figures.

The four examples shown provide a range of characteristics within a vast, binary plus planet, parameter space. The model planet in each of the four sample niches has the same eccentricity as Earth. The resonances shown as circles in Figure 1 are $\left(P_{\text {planet }}: P_{\text {binary }}\right)=2: 1,3: 1$, and so on moving out from the binary. The left two panels, Niches 4 and 8 have planets just outside the runaway greenhouse limit. The right two panels, Niches 6 and 11, have planets placed in the 
continuous BHZ. The bottom right panel, Niche 11 is limited by dynamical stability to planets far in the habitable zone. In Figure 2 the evolution of the BHZ is shown as a function of planetary semimajor axis. The planet in Niche 4 (top left) is not in the continuous BHZ, yet it has a habitability lifetime of nearly 9 Gyr. The top two panels, The sample planets in Niches 8, and 11 (bottom left and right) remain in the $\mathrm{BHZ}$ for the age of the universe.
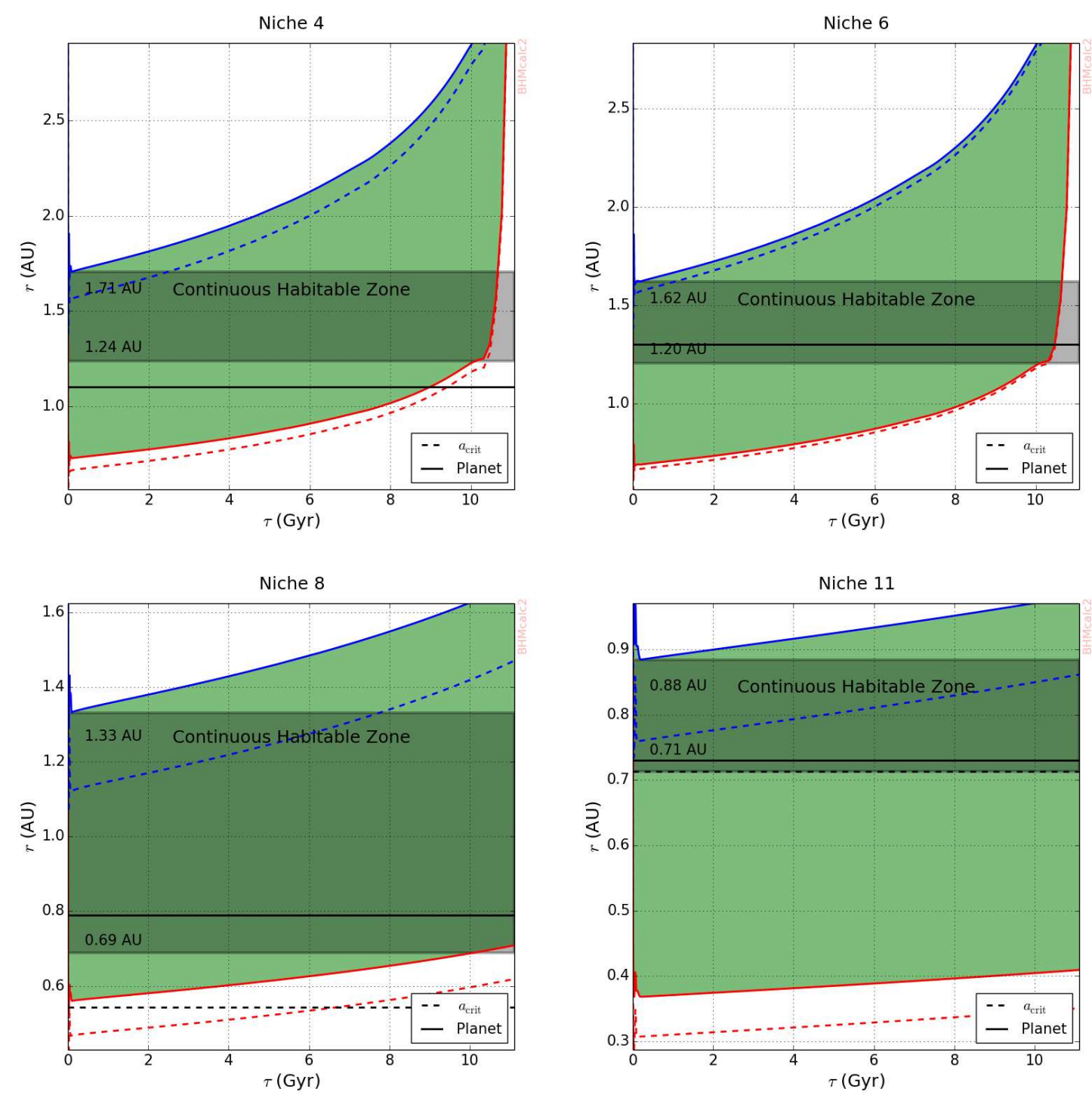

Figure 2: Continuous circumbinary habitable zones for the four mock binaries.

\subsection{Insolation and PPFD}

Photosynthetic photon flux density (hereafter PPFD) is defined as the number of photons incident per unit time on a unit surface area at the top of the atmosphere within some wavelength 
range considered to be available for use by photosynthetic life. Here we consider a PPFD bandpass of 400-1400 nm as in (23). A large bandpass is selected as long wavelengths may be harvested by life. See $(15 ; 14)$ for detailed studies of this topic.

In Figure 3, the insolation and PPFD is shown for each of the four binaries. Shaded regions indicate the extreme edges of the BHZ. Variability is increased for planets close to the binary, while near the outer edge of the BHZ it is at a minimum. For other cases, including the Kepler binaries, see the BHM calculator where examples are cataloged. As long as the planet remains within the $\mathrm{BHZ}$ at all times, variability of insolation and PPFD will remain within the limits given by the examples shown. In some cases, the additional PPFD provided by the lower mass stars will supply increased energy to the biosphere over that available for Earth life. That is, an Earth-like planet might be expected to generate significantly more biomass than the Earth.

\subsection{The Effects of Stellar Aggression}

In order to access a potential circumbinary binary for habitability stellar aggression is estimated. It is most convenient to compare the environment with the level of aggression experienced by Earth. Two proxies are employed: (1) the XUV flux, i.e. the combined flux in extreme ultraviolet and in X-rays (1-100 nm) and (2) the stellar wind (SW) particle flux. High levels of XUV flux cause non-thermal and thermal mass-loss from planetary atmospheres (see 30). In addition, high SW particle fluxes may remove tens to hundreds of bars from unmagnetized Earth-like planets $(34 ; 19 ; 20)$.

\subsubsection{Rotational Evolution}

Stellar angular momentum models including both single star mass loss evolution, (1), and tidal torques (11) are employed. Stars are modeled with two sections, a radiative core and a convective layer. Each of the three Kepler binaries with BHZ planets are analyzed with these methods by (35). Independent age estimates and primary rotation period measurements for KIC 9632895 (32), Kepler-16 (33), and Kepler-47 (25), are in good agreement with the rotation curve from the binary rotational evolution model.

In Figure 4, the rotational evolution of the stars composing the four binaries is shown. Along with the rotational period determined by the combined mass-loss and tidal braking model, the neglected-tidal solution and the orbital period of the binary are shown as dashed lines. Importantly, the pseudo-synchronous period, where the stars tend asymptotically towards is shown as a solid horizontal line.

\subsubsection{Evolution of XUV Flux}

Stellar XUV luminosity depends on chromospheric and coronal activity, which in turn is a function of rotation. Magnetic activity is estimated from stellar properties including, mass, radius, luminosity, rotation period, and metallicity using the models of (2). Since rotation of single main sequence stars slows down with age, XUV luminosity should also decrease with time (5). The rotational aging mechanism will reduce XUV luminosities and their potential harmful effects on planetary atmospheres (22).

Since most of the aggression occurs during the earliest phases of stellar evolution, the timeintegrated XUV and SW fluxes may reach an asymptotic value within the first Gyr or so for long 

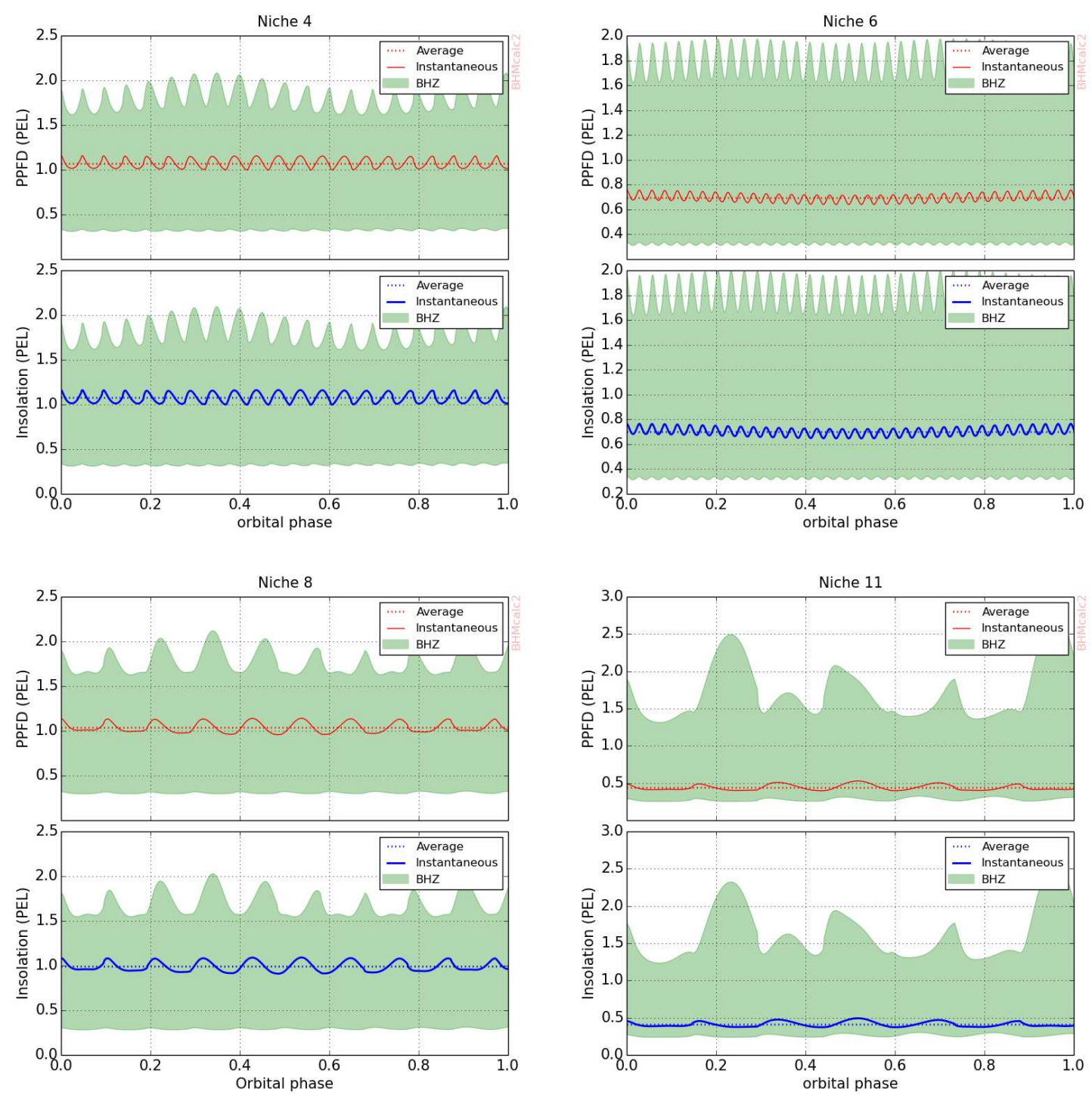

Figure 3: The insolation (bottom sub panels) and PPFD (top sub panels) is shown for Niches 4, 6, 8, and 11. The shaded region defines the limits of the BHZ, notice the dramatic differences in variability.

period binaries. However, for binaries with periods less than $\sim 20$ days or with high eccentricities XUV flux may substantially increase at late times. In Figure 5, the evolution of XUV flux incident on each planet and at the limits of the BHZ are shown with solar system limits for comparison. Notice the increase in XUV flux at late times in these cases.

The key to long term habitability for each of these model planets rests with their ability to endure increasing levels of XUV flux. This occurs after the stars evolve towards the binary period or, if eccentric, the pseudo-synchronous period. The chances of avoiding atmospheric mass-loss due to SW flux is more promising. 

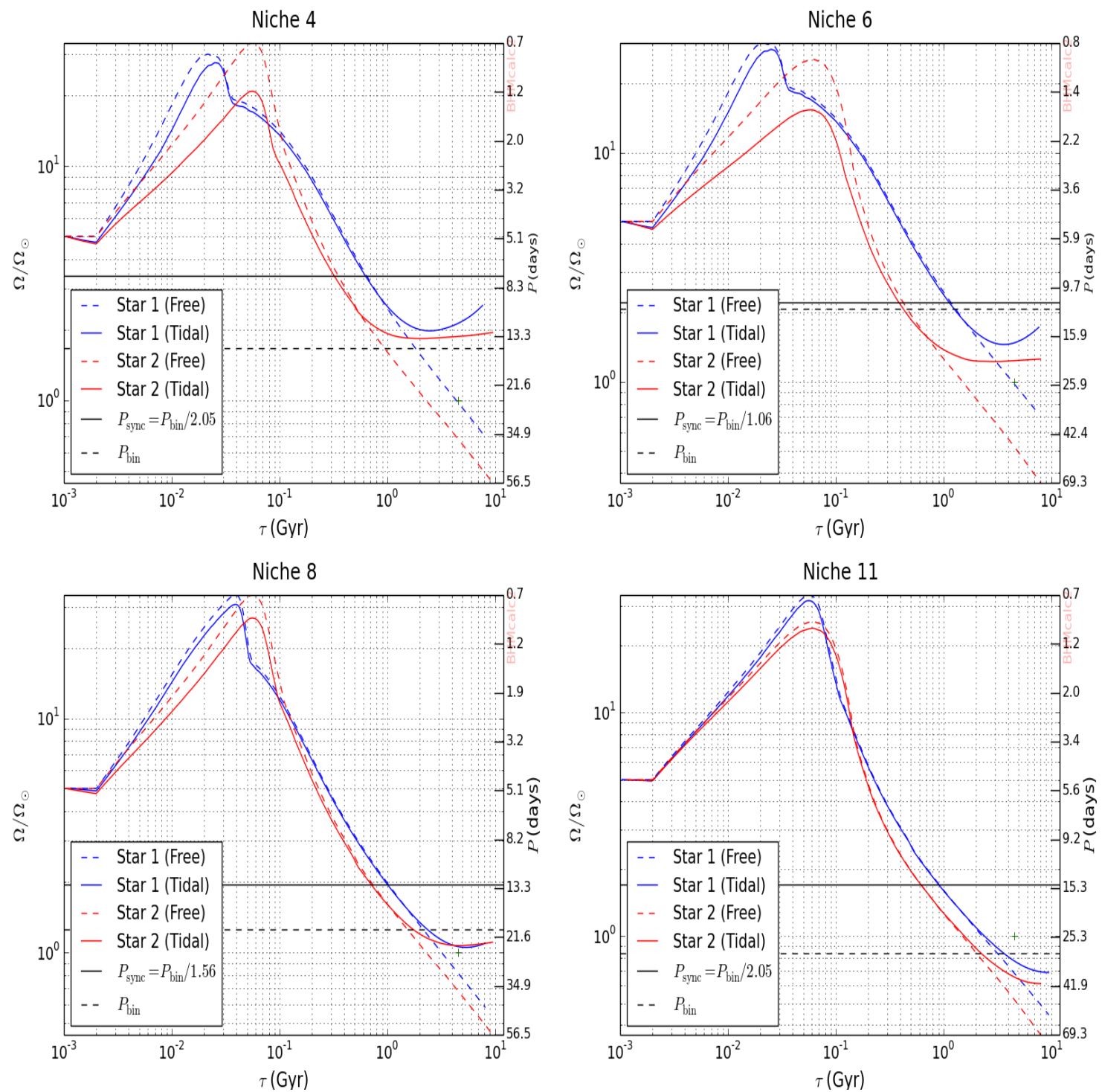

Figure 4: The rotational evolution is shown. Solid lines indicate rotational evolution the primary (blue) and secondary (red) stars. Dashed lines are for calculations neglecting tidal interaction. The binary period and pseudo-synchronous periods are shown as horizontal lines.

\subsubsection{Integrated Stellar Wind (SW) Flux}

Stellar wind properties are estimated for the purpose of angular momentum evolution. Then the derived rotational evolution translates into stellar wind evolution. A variety of parameters derived from SW evolution, integrated flux and mass loss are performed by BHMCalc. SW flux is shown in Figure 6.

\section{Discussion and Conclusions}

Although a comprehensive parameter search is beyond the scope of the current paper, we find 

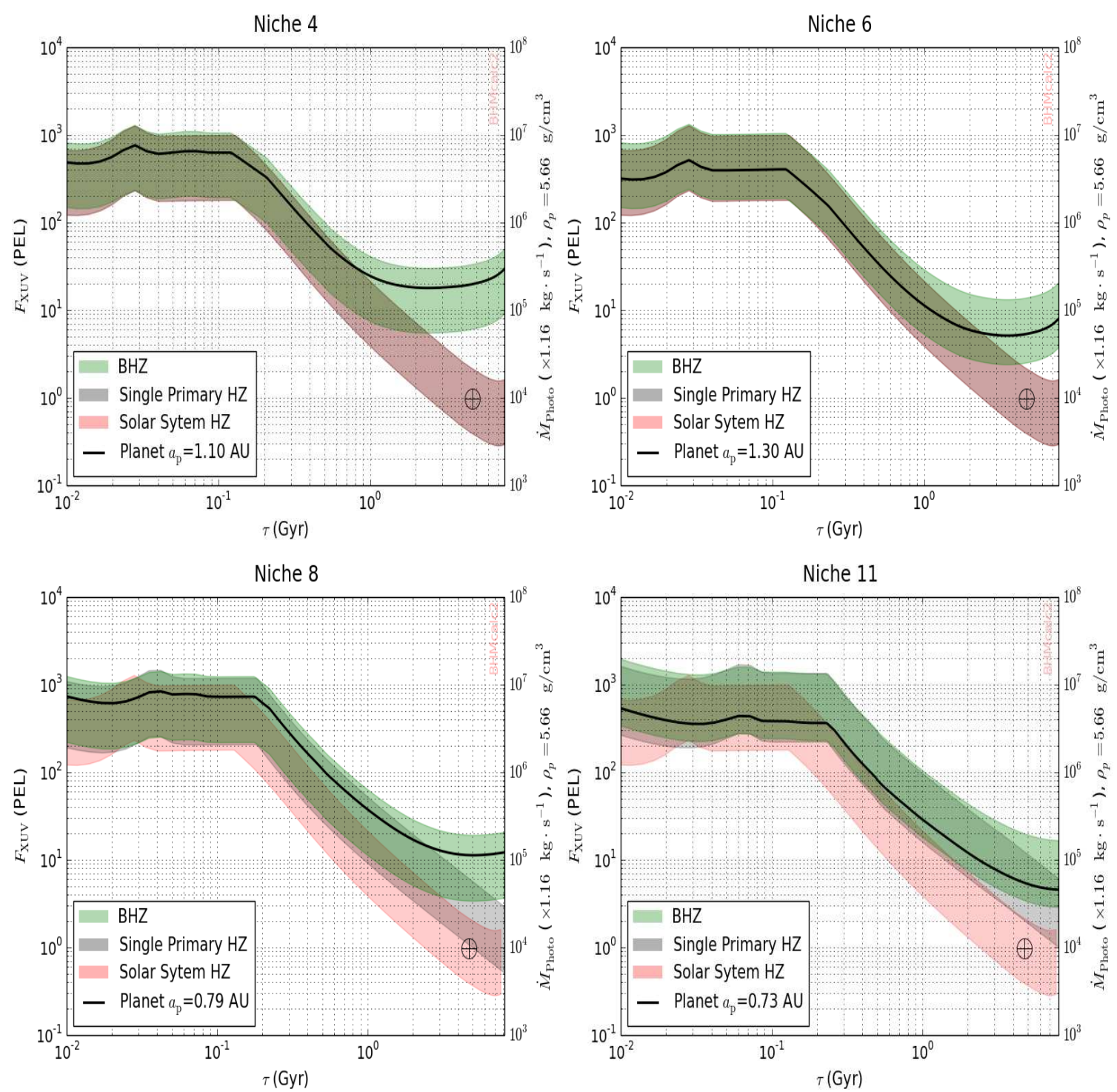

Figure 5: The evolution of XUV radiation incident on the planet is shown for the four niche binaries. Shaded areas show the BHZ (green) and solar system (gray) limits. Lower panels also show the case for a single star with the mass of the primary, dashed lines.

that BHM allows superlative BHZs in many cases, especially solar twins. In (23), we identify factors which may enable habitability in BHM protected planetary systems. These include 1) increased water retention (allowing lower mass planets), 2) multiple habitable circumbinary planets (due to wide and distant CHZs in some cases), 3) extended habitability lifetimes (for lower mass primaries), and 4) high photosynthetic photon flux density (for biomass production).

Since rotation is closely correlated to chromospheric activity, a star whose rotation has been sufficiently reduced will be less aggressive, in terms of XUV and SW flux. Hence, there will be a corresponding reduction in planetary atmospheric mass loss. Our results suggest that planets will 

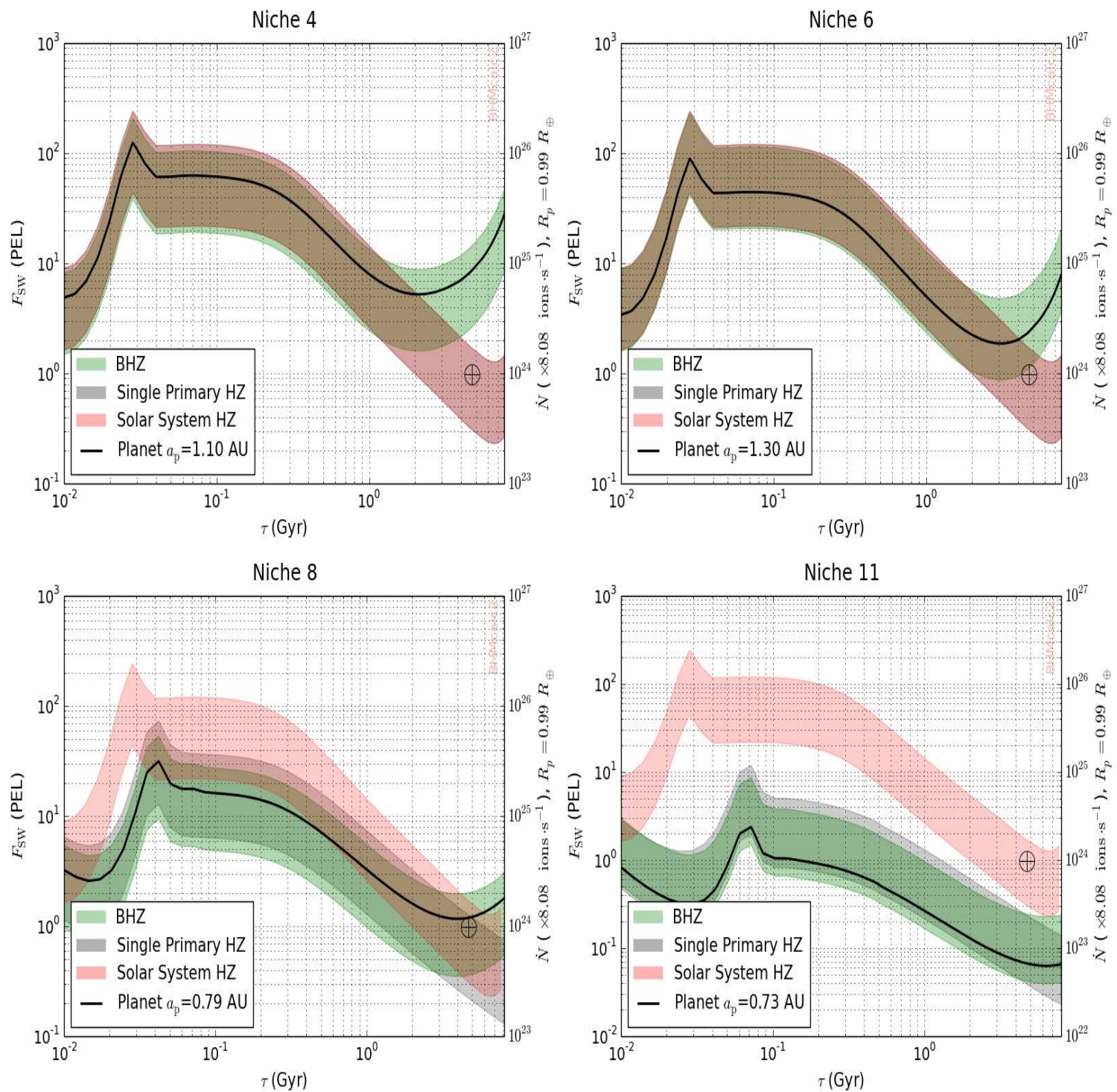

Figure 6: Binary stellar wind (SW) flux incident on the planet is shown. Shading follows the pattern of Figure 5. Niches 8 and 11 in the lower two panels have remarkably low SW flux everywhere in the BHZ.

find better conditions to maintain life when the BHM operates, as compared to single star systems. However, if the pseudo-synchronous period is too short, XUV flux may impact habitability late.

Some of the best habitability candidates are planets orbiting binary stars precisely because these planets may retain surface water. It remains to be determined if binaries actually harbor planetary systems with one or more habitable planet. If so, this increases our chances to find habitable planets in the Galaxy. Binary stars with primaries that are less that one solar mass are very long lived and plentiful. Some binaries provide high quality and quantity photons for possible use by photosynthetic life. We suggest that even moons of circumbinary planets may be habitable, see (8) for a comprehensive study of the habitability of moons with single star hosts. With plans for new 
searches for habitable exoplanets underway, we argue that circumbinary planets offer promising targets.

\section{Acknowledgments}

We thank W. Welsh, N. Haghighapor, G. Torres, S. Cranmer and A. Claret for useful discussions and insightful comments regarding the models applied in the calculator. I. Baraffe provided several key results for modeling the stellar rotation evolution of low mass-stars. J.I. Zuluaga thanks the Harvard-Smithsonian Center for Astrophysics for their hospitality during a stay in the fall/winter 2014-2015 where most of the major improvements of the calculator were achieved. Thanks to Prof. Dimitar Sasselov for this invitation under the financial support of the Fulbright Commission, Colombia, the Vicerrectoria de Docencia / Facultad de Ciencias Exactas y Naturales / Instituto de Fisica of the University of Antioquia and the program of Sostenibilidad/CODI/UdeA. Special thanks to Steffen Christensen for testing the tool and finding several major bugs. J.I. Zuluaga and P.A. Cuartas-Restrepo are supported by CODI-UdeA. P.A. Mason is supported by NMSUDACC. FACom group is supported by Estrategia de Sostenibilidad 2014-2015 UdeA.

\section{References}

[1] B. Chaboyer, P. Demarque, and M. H. Pinsonneault, Stellar models with microscopic diffusion and rotational mixing. 1: Application to the Sun, ApJ441 (1995), 865-875.

[2] S. R. Cranmer and S. H. Saar, Testing a Predictive Theoretical Model for the Mass Loss Rates of Cool Stars, ApJ741 (2011), 54.

[3] M. Cuntz and R. Bruntz, BinHab: A Numerical Tool for the Calculation of S/P-Type Habitable Zones in Binary Systems, ArXiv e-prints (2014).

[4] L. R. Doyle, J. A. Carter, D. C. Fabrycky, R. W. Slawson, S. B. Howell, J. N. Winn, J. A. Orosz, A. Prsa, W. F. Welsh, S. N. Quinn, D. Latham, G. Torres, L. A. Buchhave, G. W. Marcy, J. J. Fortney, A. Shporer, E. B. Ford, J. J. Lissauer, D. Ragozzine, M. Rucker, N. Batalha, J. M. Jenkins, W. J. Borucki, D. Koch, C. K. Middour, J. R. Hall, S. McCauliff, M. N. Fanelli, E. V. Quintana, M. J. Holman, D. A. Caldwell, M. Still, R. P. Stefanik, W. R. Brown, G. A. Esquerdo, S. Tang, G. Furesz, J. C. Geary, P. Berlind, M. L. Calkins, D. R. Short, J. H. Steffen, D. Sasselov, E. W. Dunham, W. D. Cochran, A. Boss, M. R. Haas, D. Buzasi, and D. Fischer, Kepler-16: A Transiting Circumbinary Planet, Science 333 (2011), 1602-.

[5] A. Garcés, S. Catalán, and I. Ribas, Time evolution of high-energy emissions of low-mass stars. I. Age determination using stellar chronology with white dwarfs in wide binaries, A\&A531 (2011), A7.

[6] N. Haghighipour and L. Kaltenegger, Calculating the Habitable Zone of Binary Star Systems. II. P-type Binaries, ApJ777 (2013), 166. 
[7] R. S. Harrington, Planetary orbits in binary stars, AJ82 (1977), 753-756.

[8] R. Heller, D. Williams, D. Kipping, M. A. Limbach, E. Turner, R. Greenberg, T. Sasaki, É. Bolmont, O. Grasset, K. Lewis, R. Barnes, and J. I. Zuluaga, Formation, Habitability, and Detection of Extrasolar Moons, Astrobiology 14 (2014), 798-835.

[9] T. C. Hinse, N. Haghighipour, V. B. Kostov, and K. Goździewski, Predicting a third planet in the Kepler-47 circumbinary system, ArXiv e-prints (2014).

[10] M. J. Holman and P. A. Wiegert, Long-Term Stability of Planets in Binary Systems, AJ117 (1999), 621-628.

[11] P. Hut, Tidal evolution in close binary systems, A\&A99 (1981), 126-140.

[12] S. R. Kane and N. R. Hinkel, On the Habitable Zones of Circumbinary Planetary Systems, ApJ762 (2013), 7.

[13] J. F. Kasting, D. P. Whitmire, and R. T. Reynolds, Habitable Zones around Main Sequence Stars, Icarus101 (1993), 108-128.

[14] N. Y. Kiang, A. Segura, G. Tinetti, Govindjee, R. E. Blankenship, M. Cohen, J. Siefert, D. Crisp, and V. S. Meadows, Spectral Signatures of Photosynthesis. II. Coevolution with Other Stars And The Atmosphere on Extrasolar Worlds, Astrobiology 7 (2007), 252-274.

[15] N. Y. Kiang, J. Siefert, Govindjee, and R. E. Blankenship, Spectral Signatures of Photosynthesis. I. Review of Earth Organisms, Astrobiology 7 (2007), 222-251.

[16] R. K. Kopparapu, R. M. Ramirez, J. SchottelKotte, J. F. Kasting, S. Domagal-Goldman, and V. Eymet, Habitable Zones around Main-sequence Stars: Dependence on Planetary Mass, ApJ787 (2014), L29.

[17] V. B. Kostov, P. R. McCullough, J. A. Carter, M. Deleuil, R. F. Díaz, D. C. Fabrycky, G. Hébrard, T. C. Hinse, T. Mazeh, J. A. Orosz, Z. I. Tsvetanov, and W. F. Welsh, Kepler413b: A Slightly Misaligned, Neptune-size Transiting Circumbinary Planet, ApJ784 (2014), 14.

[18] V. B. Kostov, P. R. McCullough, T. C. Hinse, Z. I. Tsvetanov, G. Hébrard, R. F. Díaz, M. Deleuil, and J. A. Valenti, A Gas Giant Circumbinary Planet Transiting the F Star Primary of the Eclipsing Binary Star KIC 4862625 and the Independent Discovery and Characterization of the Two Transiting Planets in the Kepler-47 System, ApJ770 (2013), 52.

[19] H. Lammer, J. F. Kasting, E. Chassefière, R. E. Johnson, Y. N. Kulikov, and F. Tian, Atmospheric Escape and Evolution of Terrestrial Planets and Satellites, p. 399, 2009.

[20] H. Lammer, H. I. M. Lichtenegger, M. L. Khodachenko, Y. N. Kulikov, and J. Griessmeier, The Loss of Nitrogen-rich Atmospheres from Earth-like Exoplanets within M-star Habitable Zones, Astronomical Society of the Pacific Conference Series (J. P. Beaulieu, S. Dieters, \& G. Tinetti, ed.), Astronomical Society of the Pacific Conference Series, vol. 450, January 2012, p. 139. 
[21] P. A. Mason and J. M. Clark, Habitability In Close Binary Systems: Conditions For An EarthAnalogue, American Astronomical Society Meeting Abstracts \#220, American Astronomical Society Meeting Abstracts, vol. 220, May 2012, p. 525.04.

[22] P. A. Mason, J. I. Zuluaga, J. M. Clark, and P. A. Cuartas-Restrepo, Rotational Synchronization May Enhance Habitability for Circumbinary Planets: Kepler Binary Case Studies, ApJ774 (2013), L26.

[23] P. A. Mason, J. I. Zuluaga, P. A. Cuartas-Restrepo, and J. M. Clark, Circumbinary Habitability Niches, ArXiv e-prints (2014).

[24] T. W. A. Müller and N. Haghighipour, Calculating the Habitable Zones of Multiple Star Systems with a New Interactive Web Site, ApJ782 (2014), 26.

[25] J. A. Orosz, W. F. Welsh, J. A. Carter, E. Brugamyer, L. A. Buchhave, W. D. Cochran, M. Endl, E. B. Ford, P. MacQueen, D. R. Short, G. Torres, G. Windmiller, E. Agol, T. Barclay, D. A. Caldwell, B. D. Clarke, L. R. Doyle, D. C. Fabrycky, J. C. Geary, N. Haghighipour, M. J. Holman, K. A. Ibrahim, J. M. Jenkins, K. Kinemuchi, J. Li, J. J. Lissauer, A. Prša, D. Ragozzine, A. Shporer, M. Still, and R. A. Wade, The Neptune-sized Circumbinary Planet Kepler-38b, ApJ758 (2012), 87.

[26] J. A. Orosz, W. F. Welsh, J. A. Carter, D. C. Fabrycky, W. D. Cochran, M. Endl, E. B. Ford, N. Haghighipour, P. J. MacQueen, T. Mazeh, R. Sanchis-Ojeda, D. R. Short, G. Torres, E. Agol, L. A. Buchhave, L. R. Doyle, H. Isaacson, J. J. Lissauer, G. W. Marcy, A. Shporer, G. Windmiller, T. Barclay, A. P. Boss, B. D. Clarke, J. Fortney, J. C. Geary, M. J. Holman, D. Huber, J. M. Jenkins, K. Kinemuchi, E. Kruse, D. Ragozzine, D. Sasselov, M. Still, P. Tenenbaum, K. Uddin, J. N. Winn, D. G. Koch, and W. J. Borucki, Kepler-47: A Transiting Circumbinary Multiplanet System, Science 337 (2012), 1511-.

[27] B. Quarles, Z. E. Musielak, and M. Cuntz, Habitability of Earth-mass Planets and Moons in the Kepler-16 System, ApJ750 (2012), 14.

[28] J. Sanz-Forcada, S. Desidera, and G. Micela, Effects of X-ray and extreme UV radiation on circumbinary planets, A\&A570 (2014), A50.

[29] M. E. Schwamb, J. A. Orosz, J. A. Carter, W. F. Welsh, D. A. Fischer, G. Torres, A. W. Howard, J. R. Crepp, W. C. Keel, C. J. Lintott, N. A. Kaib, D. Terrell, R. Gagliano, K. J. Jek, M. Parrish, A. M. Smith, S. Lynn, R. J. Simpson, M. J. Giguere, and K. Schawinski, Planet Hunters: A Transiting Circumbinary Planet in a Quadruple Star System, ApJ768 (2013), 127.

[30] F. Tian, Thermal Escape from Super Earth Atmospheres in the Habitable Zones of M Stars, ApJ703 (2009), 905-909.

[31] W. F. Welsh, J. A. Orosz, J. A. Carter, D. C. Fabrycky, E. B. Ford, J. J. Lissauer, A. Prša, S. N. Quinn, D. Ragozzine, D. R. Short, G. Torres, J. N. Winn, L. R. Doyle, T. Barclay, N. Batalha, S. Bloemen, E. Brugamyer, L. A. Buchhave, C. Caldwell, D. A. Caldwell, J. L. Christiansen, D. R. Ciardi, W. D. Cochran, M. Endl, J. J. Fortney, T. N. Gautier, III, R. L. Gilliland, M. R. 
Haas, J. R. Hall, M. J. Holman, A. W. Howard, S. B. Howell, H. Isaacson, J. M. Jenkins, T. C. Klaus, D. W. Latham, J. Li, G. W. Marcy, T. Mazeh, E. V. Quintana, P. Robertson, A. Shporer, J. H. Steffen, G. Windmiller, D. G. Koch, and W. J. Borucki, Transiting circumbinary planets Kepler-34 b and Kepler-35 b, Nature481 (2012), 475-479.

[32] W. F. Welsh, J. A. Orosz, D. R. Short, N. Haghighipour, L. A. Buchhave, L. R. Doyle, D. C. Fabrycky, T. C. Hinse, S. Kane, V. Kostov, T. Mazeh, S. M. Mills, T. W. A. Mueller, B. Quarles, S. N. Quinn, D. Ragozzine, A. Shporer, J. H. Steffen, L. Tal-Or, G. Torres, G. Windmiller, and W. J. Borucki, KIC 9632895 - The 10th Kepler Transiting Circumbinary Planet, ArXiv e-prints (2014).

[33] J. N. Winn, S. Albrecht, J. A. Johnson, G. Torres, W. D. Cochran, G. W. Marcy, A. W. Howard, H. Isaacson, D. Fischer, L. Doyle, W. Welsh, J. A. Carter, D. C. Fabrycky, D. Ragozzine, S. N. Quinn, A. Shporer, S. B. Howell, D. W. Latham, J. Orosz, A. Prsa, R. W. Slawson, W. J. Borucki, D. Koch, T. Barclay, A. P. Boss, J. Christensen-Dalsgaard, F. R. Girouard, J. Jenkins, T. C. Klaus, S. Meibom, R. L. Morris, D. Sasselov, M. Still, and J. Van Cleve, Spin-Orbit Alignment for the Circumbinary Planet Host Kepler-16 A, ApJ741 (2011), L1.

[34] J. Zendejas, A. Segura, and A. C. Raga, Atmospheric mass loss by stellar wind from planets around main sequence M stars, Icarus210 (2010), 539-544.

[35] J. I. Zuluaga, P. A. Mason, and P. A. Cuartas, Constraining the Radiation and Plasma Environment of the Kepler Circumbinary Habitable Zone Planets, ArXiv e-prints (2015). 